\title{
ON MULTIVARIATE PANJER RECURSIONS
}

\author{
BY \\ BJøRN SUNDT \\ University of Bergen \& University of Melbourne
}

\begin{abstract}
In the present paper we generalise Panjer's (1981) recursion for compound distributions to a multivariate situation where each claim event generates a random vector. We discuss situations within insurance where such models could be applicable, and consider some special cases of the general algorithm. Finally we deduce from the algorithm a multivariate extension of De Pril's (1985) recursion for convolutions.
\end{abstract}

\section{INTRODUCTION}

1A. Let $N$ denote the number of claims occurring in an insurance portfolio within a given period, and $U_{i}$ the amount of the $i$ th of these claims. We assume that these claim amounts are positive, integer-valued, mutually independent and identically distributed with common probability function $f$, and independent of $N$. Let $p$ denote the probability function of $N$. Then the distribution of the aggregate claims $X=\sum_{i=1}^{N} U_{i}$ is a compound distribution with probability function

$$
g=\sum_{n=0}^{\infty} p(n) f^{n *}
$$

Panjer (1981) presented an algorithm for recursive evaluation of $g$ when $p$ satisfies a recursion in the form

$$
p(n)=\left(a+\frac{b}{n}\right) p(n-1) . \quad(n=1,2, \ldots)
$$

We obtain that $N$ has a Poisson distribution when $a=0$, a negative binomial distribution when $a>0$, and a binomial distribution when $a<0$. Panjer's paper motivated the development of an extensive theory on recursive methods for compound distributions as well as other types of distributions that would be appropriate for aggregate claims of insurance portfolios. 
1B. Hesselager (1996) presented some bivariate extensions of Panjer's recursion, using bivariate generalisations of the counting distribution. $\mathrm{He}$ considered a situation with two portfolios. For $j=1,2$, let $N_{j}$ denote the number of claims in portfolio $j$ and $W_{i j}$ the amount of the $i$ th of these claims. We assume that the $W_{i j}$ 's are positive, integer-valued, mutually independent, independent of the claim numbers, and for fixed $j$ identically distributed with common probability function $f_{j}$. The aggregate claim amount from portfolio $j$ is $X_{j}=\sum_{i=1}^{N_{j}} W_{i j}$. Let $q$ denote the joint probability function of $N_{1}$ and $N_{2}$ and $g$ the joint probability function of $X_{1}$ and $X_{2}$. Then

$$
g\left(x_{i}, x_{2}\right)=\sum_{n_{1}=0}^{x_{1}} \sum_{n_{2}=0}^{x_{2}} q\left(n_{1}, n_{2}\right) f_{1}^{n_{1 *}}\left(x_{1}\right) f_{2}^{n_{2^{*}}}\left(x_{2}\right) . \quad\left(x_{1}, x_{2}=0,1, \ldots\right)
$$

Hesselager deduced recursions for $g$ in some cases where $q$ can be interpreted as a bivariate extension of Panjer's counting distributions.

1C. In the present paper we shall study a multivariate extension of Panjer's recursion in another direction than Hesselager. We shall still assume that the claim number is one-dimensional, and that its distribution satisfies (1.2). However, we now assume that each claim is an $m$-dimensional random vector, and that these vectors are mutually independent and identically distributed and independent of the number of claims. This can be interpreted as if the number of claims is now the number of claim events within a portfolio of $m$ policies and the severity vector represents the vector of payments to each policy caused by one claim event. We shall motivate this model further in Section 2.

After the motivation in Section 2 we deduce the main result in Section 3. In Section 4 we discuss some special cases. Finally, in Section 5, we look at an additional special case, from which we deduce a multivariate extension of De Pril's (1985) recursion for convolutions.

\section{Motivation}

2A. Let $N$ denote the number of claim events,

$$
\mathbf{U}_{i}=\left(U_{i 1}, \ldots, U_{i m}\right)^{\prime} \quad(i=1,2, \ldots)
$$

an $m$-dimensional vector generated by the $i$ th of these events, and

$$
\mathbf{X}=\left(X_{1}, \ldots, X_{m}\right)^{\prime}=\sum_{i=1}^{N} \mathbf{U}_{i}
$$


(we interpret $\sum_{i=c}^{d}=0$ when $d<c$ ). We assume that $\mathbf{U}_{1}, \mathbf{U}_{2}, \ldots$ are mutually independent and identically distributed with probability function $f$, and independent of $N$. It is further assumed that all the $U_{i j}$ 's are non-negative. Let $p$ and $g$ denote the probability functions of $N$ and $\mathbf{X}$ respectively. Then (1.1) still holds.

2B. In this subsection we shall indicate some situations where our model could be appropriate.

1. As indicated in Section 1, in a portfolio with $m$ policies we can interpret $U_{i j}$ as the claim amount of policy $j$ caused by claim event $i$. A natural example is windstorm insurance where one windstorm could affect more than one policy. The variable $X_{j}$ represents the aggregate claims of policy $j$.

2. If we let $U_{i j}$ be equal to one if claim event $i$ causes a payment on policy $j$, and zero otherwise, then $X_{j}$ will be the number of claims of policy $j$. Analogously we can develop multivariate counting distributions in the following examples.

3. Another application of the model would be to a situation where each claim event can induce various types of claims. These types could have different reinsurance covers. Let $m$ be the number of types and $U_{i j}$ the payment of type $j$ at claim event $i$. Then $X_{j}$ represents the aggregate claims of type $j$. We assume that $X_{j}$ is covered by a reinsurance such that the insurance company retains $r_{j}\left(X_{j}\right)$. Thus the total aggregate claims will be $Z=\sum_{j=1}^{m} r_{j}\left(X_{j}\right)$. From $g$ we can evaluate the distribution of $Z$. Perhaps $Z$ is covered by an umbrella cover. Then we can apply the distribution of $Z$ to evaluate the premium for the umbrella. A good example of an insurance class where different types of claims could have different reinsurance covers, is motor insurance, where the reinsurance would often be different for vehicle damage and personal injury. Analogously, in workers' compensation insurance one could have different reinsurance for sickness and accident.

4. We now return to the case with only one type of claim and let $m=2$. Of claim $i$ the ceding company retains $U_{i 1}$, and the reinsurer covers the rest, $U_{i 2}$. Then $X_{1}$ and $X_{2}$ represent the total payments of the insurer and the reinsurer respectively, and by our generalised Panjer recursion we can evaluate their joint distribution. In subsection $4 \mathrm{H}$ we shall study this situation in the special case of unlimited excess-of-loss reinsurance.

5. Let us now consider the run-off of the claims incurred in an insurance portfolio during a specified year. We assume that all claims will have been settled after $m$ years. Let $U_{i j}$ be the part of the $i$ th claim paid in development year $j$. Then $X_{j}$ will be the total payments in development year $j$.

2C. By conditioning on $N$ we easily obtain that

$$
\operatorname{Cov}\left(X_{j}, X_{k}\right)=\mathrm{E} N \operatorname{Cov}\left(U_{1 j}, U_{1 k}\right)+\mathrm{E} U_{1 j} \mathrm{E} U_{1 k} \operatorname{Var} N .
$$


If $U_{1 j}$ and $U_{1 k}$ are independent, then (2.1) gives

$$
\operatorname{Cov}\left(X_{j}, X_{k}\right)=\mathrm{E} U_{1 j} \mathrm{E} U_{1 k} \operatorname{Var} N>0, \quad(k \neq j)
$$

that is, because $N$ affects all the policies, the aggregate claims of different policies will be positively correlated when the severities of different policies are independent.

We rewrite $(2.1)$ as

$$
\operatorname{Cov}\left(X_{j}, X_{k}\right)=\mathrm{E} N \mathrm{E} U_{1 j} U_{1 k}+\mathrm{E} U_{1 j} \mathrm{E} U_{1 k}(\operatorname{Var} N-\mathrm{E} N) .
$$

If $N$ is Poisson distributed, then $\operatorname{Var} N=\mathrm{E} N$, and we obtain

$$
\operatorname{Cov}\left(X_{j}, X_{k}\right)=\mathrm{E} N \mathrm{E} U_{1 j} U_{1 k} \text {. }
$$

We see that in this case, for $k \neq j, X_{j}$ and $X_{k}$ are uncorrelated if and only if $\mathrm{E} U_{1 j} U_{1 k}=0$. This implies that $U_{1 j}$ and $U_{1 k}$ cannot both be positive. In the situation where $U_{i j}$ denotes the amount caused to policy $j$ by claim event $i$, this means that a claim event cannot hit more than one policy. In the situation where $U_{i j}$ denotes the amount of type $j$ caused by one claim, it means that a claim cannot cause payments of more than one type; there cannot at the same time be payments on death and disability. In fact, in this case we have not only that $X_{j}$ and $X_{k}$ are uncorrelated, but they are even independent, cf. e.g. Sundt (1993).

Leaving the Poisson assumption, but keeping the assumption that $\mathrm{E} U_{1 j} U_{1 k}=0$ for $k \neq j$, we obtain

$$
\operatorname{Cov}\left(X_{j}, X_{k}\right)=\mathrm{E} U_{1 j} \mathrm{E} U_{1 k}(\operatorname{Var} N-\mathrm{E} N) . \quad(k \neq j)
$$

When $N$ is negative binomially distributed, we have $\operatorname{Var} N>\mathrm{E} N$, and thus $X_{j}$ and $X_{k}$ are positively correlated. On the other hand, if $N$ is binomially distributed, then $\operatorname{Var} N<\mathrm{E} N$, and $X_{j}$ and $X_{k}$ are negatively correlated.

\section{MAIN RESUlt}

3A. In the following we shall apply the notation

$$
\begin{aligned}
\mathbf{x}=\left(x_{1}, \ldots, x_{m}\right)^{\prime} ; & \mathbf{u}=\left(u_{1}, \ldots, u_{m}\right)^{\prime} \\
x .=\sum_{j=1}^{m} x_{j} ; & \text { u. }=\sum_{j=1}^{m} u_{j} .
\end{aligned}
$$

We shall always tacitly assume that the elements of $\mathbf{x}$ and $\mathbf{u}$ are non-negative integers. By $\mathbf{u} \leq \mathbf{x}$ we shall mean that $u_{j} \leq x_{j}$ for $j=1, \ldots, m$ and by $\mathbf{u}<\mathbf{x}$ that $u_{j} \leq x_{j}$ for $j=1, \ldots, m$ with strict inequality for at least one $j$. For $j=1, \cdots, m$ we define $\mathbf{e}_{j}$ to be the $m \times 1$ vector whose $j$ th element is 1 and all the other elements are 0 . 
Under the additional assumption that $p$ satisfies (1.2), we shall deduce multivariate extensions of Panjer's recursion.

Theorem 1. The probability function $g$ satisfies the recursion

$$
\begin{gathered}
g(\mathbf{0})=\sum_{n=0}^{\infty} p(n) f(\mathbf{0})^{n} \\
x_{k} g(\mathbf{x})=\frac{1}{1-a f(\mathbf{0})} \sum_{0<\mathbf{u} \leq \mathbf{x}}\left(a x_{k}+b u_{k}\right) f(\mathbf{u}) g(\mathbf{x}-\mathbf{u}) \quad(\mathbf{x}>\mathbf{0})
\end{gathered}
$$

for $k=1,2, \ldots, m$.

Proof. Formula (3.1) follows from (1.1) and the assumption that $\sum_{j=1}^{m} U_{1 j}$ is non-negative.

When $\mathbf{x}>\mathbf{0}$, we have

$$
\begin{aligned}
& x_{k} g(\mathbf{x})=\sum_{n=1}^{\infty} x_{k} p(n) f^{n^{*}}(\mathbf{x})=\sum_{n=1}^{\infty} x_{k} p(n-1)\left(a+\frac{b}{n}\right) f^{n^{*}}(\mathbf{x})= \\
& \sum_{n=1}^{\infty} p(n-1) \mathrm{E}\left[a x_{k}+b U_{1 k} \mid \sum_{i=1}^{n} \mathbf{U}_{i}=\mathbf{x}\right] f^{n^{*}}(\mathbf{x})= \\
& \sum_{n=1}^{\infty} p(n-1) \sum_{0 \leq \mathbf{u} \leq \mathbf{x}}\left(a x_{k}+b u_{k}\right) f(\mathbf{u}) f^{(n-1)^{*}}(\mathbf{x}-\mathbf{u})= \\
& \sum_{\mathbf{0} \leq \mathbf{u} \leq \mathbf{x}}\left(a x_{k}+b u_{k}\right) f(\mathbf{u}) \sum_{n=1}^{\infty} p(n-1) f^{(n-1)^{*}}(\mathbf{x}-\mathbf{u})= \\
& \sum_{\mathbf{0} \leq \mathbf{u} \leq \mathbf{x}}\left(a x_{k}+b u_{k}\right) f(\mathbf{u}) g(\mathbf{x}-\mathbf{u})= \\
& a x_{k} f(\mathbf{0}) g(\mathbf{x})+\sum_{\mathbf{0} \leq \mathbf{u} \leq \mathbf{x}}\left(a x_{k}+b u_{k}\right) f(\mathbf{u}) g(\mathbf{x}-\mathbf{u}),
\end{aligned}
$$

and solving for $x_{k} g(\mathbf{x})$ gives (3.2).

This completes the proof of Theorem 1.

Q.E.D.

When $x_{k}>0$, we can divide (3.2) by $x_{k}$. We obtain

$$
g(\mathbf{x})=\frac{1}{1-a f(\mathbf{0})} \sum_{\mathbf{0}<\mathbf{u} \leq \mathbf{x}}\left(a+b \frac{u_{k}}{x_{k}}\right) f(\mathbf{u}) g(\mathbf{x}-\mathbf{u}), \quad\left(\mathbf{x} \geq \mathbf{e}_{k}\right)
$$

which together with (3.1) can be applied for recursive evaluation of $g$. 
When $f(0)=0,(3.1)$ and (3.3) reduce to

$$
\begin{gathered}
g(\mathbf{0})=p(0) \\
g(\mathbf{x}) \sum_{\mathbf{0}<\mathbf{u} \leq \mathbf{x}}\left(a+b \frac{u_{k}}{x_{k}}\right) f(\mathbf{u}) g(\mathbf{x}-\mathbf{u}), \quad\left(\mathbf{x} \geq \mathbf{e}_{k}\right)
\end{gathered}
$$

In the situation with a portfolio with $m$ policies, this assumption means that any claim event will affect at least one policy. When $m=1,(3.4)$ reduces to

$$
g(x)=\sum_{u=1}^{x}\left(a+b \frac{u}{x}\right) f(u) g(x-u) . \quad(x=1,2, \ldots)
$$

This is the recursion deduced by Panjer (1981).

3B. It is interesting to notice that when $b \neq 0$, the recursion (3.3) is not symmetric in the policies; one of them, policy $k$, is treated in a special way. In practice it seems to be computationally most efficient to choose the policy where the claim amount can take the least number of values. We shall return to this in Section 4.

Also, we see that we have to apply another policy than policy $k$ as the special policy when $x_{k}=0$. Let us apply policy $l$ when $x_{k}=0$ and $x_{l}>0$. However, when $x_{k}=x_{l}=0$, we have to apply a third policy as the special policy, and so on. In the worst case, we will have to involve each of the $m$ policies as the special policy at some stage. This may make the recursion of Theorem 1 awkward to program. However, it will normally involve less arithmetic operations than brute force evaluation by (1.1).

In some cases the problem of $x_{k}=0$ is reduced or vanishes completely. We see that the problem arises when $x_{k}=0$ and $x_{j}>0$ for at least one $j \neq k$. If $U_{i k}$ is always positive when at least one of the other $U_{i j}$ 's is positive, then $X_{k}$ is also positive only when at least one of the other $X_{j}$ 's is positive. Therefore $g(\mathbf{x})=0$ when $x_{k}=0$ and some other $x_{j}$ is positive, and the case $x_{k}=0$ does not create any problem for the recursion. In the situation with excess-of-loss reinsurance mentioned in Example 4 in subsection 2B the ceding company will always make payments for own account when there are positive reinsurance payments, and thus, with $k=1$, the case $x_{k}=0$ is unproblematic. We shall return to this situation in subsection $4 \mathrm{H}$.

It seems that in practice the multivariate recursions would be applicable only when $m$ is small, as otherwise the computational work would be prohibitive.

3C. Let $\mathbf{c}=\left(c_{1}, \ldots, c_{m}\right)^{\prime}$ be a constant vector. Multiplication of (3.2) by $c_{k}$ and summation over $k$ gives

$$
g(\mathbf{x}) \mathbf{c}^{\prime} \mathbf{x}=\frac{1}{1-a f(\mathbf{0})} \sum_{0<\mathbf{u} \leq \mathbf{x}}\left(a \mathbf{c}^{\prime} \mathbf{x}+b \mathbf{c}^{\prime} \mathbf{u}\right) f(\mathbf{u}) g(\mathbf{x}-\mathbf{u}) . \quad(x>0)
$$


When $\mathbf{c}^{\prime} \mathbf{x} \neq 0$, we can divide (3.6) by $\mathbf{c}^{\prime} \mathbf{x}$, and we obtain

$$
g(\mathbf{x})=\frac{1}{1-a f(0)} \sum_{\mathbf{0}<\mathbf{u} \leq \mathbf{x}}\left(a+b \frac{\mathbf{c}^{\prime} \mathbf{u}}{\mathbf{c}^{\prime} \mathbf{x}}\right) f(\mathbf{u}) g(\mathbf{x}-\mathbf{u}) . \quad\left(\mathbf{c}^{\prime} \mathbf{x} \neq \mathbf{0}\right)
$$

Normally we would choose the $c_{j}$ 's equal to zero or one. In particular, when $\mathbf{c}=\mathbf{e}_{k}$ (3.7) reduces to (3.3). Another interesting case is when $c_{j}=1$ for all $j$. Then $\mathbf{c}^{\prime} \mathbf{x}=x$., which is always positive when $\mathbf{x}>\mathbf{0}$. Thus

$$
g(\mathbf{x})=\frac{1}{1-a f(0)} \sum_{0<\mathbf{u} \leq \mathbf{x}}\left(a+b \frac{u}{x}\right) f(\mathbf{u}) g(\mathbf{x}-\mathbf{u}) . \quad(\mathbf{x}>\mathbf{0})
$$

Under efficient programming, (3.8) is not necessarily significantly more timeconsuming than (3.3), and it has the advantage that it can be applied for all $\mathbf{x}>\mathbf{0}$.

When $m=1$, the recursion (3.8) reduces to Panjer's recursion (3.5).

For the rest of the paper we shall mainly concentrate on recursions with division by $x_{k}$ and leave to the readers to deduce corresponding recursions with division by $x$. or $\mathbf{c}^{\prime} \mathbf{x}$.

3D. The way we extended Panjer's univariate recursion, can easily be applied to other univariate recursions. As an example, let us generalise the recursion (3.3) to the situation when $p$ satisfies a recursion

$$
p(n)=\sum_{s=1}^{r}\left(a_{s}+\frac{b_{s}}{n}\right) p(n-s) \quad(n=1,2, \ldots)
$$

for some positive integer $r$; we have $p(n)=0$ for $n<0$. When $r=1$, (3.9) reduces to (1.2). By modifying the proof of Theorem 9 in Sundt (1992) analogous to the way we modified the proof of Theorem 10.6 in Sundt (1993) for the proof of Theorem 1, we obtain

$g(\mathbf{x})=\frac{1}{1-\sum_{s=1}^{r} a_{s} f(\mathbf{0})^{s}} \sum_{\mathbf{0}<\mathbf{u} \leq \mathbf{x}} g(\mathbf{x}-\mathbf{u}) \sum_{s=1}^{r}\left(a_{s}+\frac{b_{s}}{s} \frac{u_{k}}{x_{k}}\right) f^{s *}(\mathbf{u}), \quad\left(\mathbf{x} \geq \mathbf{e}_{k}\right)$

and analogous to (3.8) we obtain

$g(\mathbf{x})=\frac{1}{1-\sum_{s=1}^{r} a_{s} f(\mathbf{0})^{s}} \sum_{\mathbf{0}<\mathbf{u} \leq \mathbf{x}} g(\mathbf{x}-\mathbf{u}) \sum_{s=1}^{r}\left(a_{s}+\frac{b_{s}}{s} \frac{u}{x .}\right) f^{s *}(\mathbf{u}) . \quad(\mathbf{x}>\mathbf{0})$

These recursions reduce to respectively (3.3) and (3.8) when $r=1$, and to the recursion of Sundt (1992) when $m=1$.

The recursions of the present subsection are further analysed in Sundt (1998). 


\section{SPECial cases}

4A. We consider the situation where a claim event can induce various types of claims, and assume that one claim event cannot induce claims of more than one type. For $j=1,2, \ldots, m$ let $U_{i j}$ be equal to one if the $i$ th claim event induces a claim of type $j$ and zero otherwise. Then $\sum_{j=1}^{m} U_{i j}=1$. Let

$$
h(j)=\operatorname{Pr}\left(U_{i j}=1\right) . \quad(j=1,2, \ldots, m)
$$

Application of (3.8) gives

$$
g(\mathbf{x})=\left(a+\frac{b}{x}\right) \sum_{j=1}^{m} h(j) g\left(\mathbf{x}-\mathbf{e}_{j}\right) . \quad(\mathbf{x}>\mathbf{0})
$$

4B. For the rest of Section 4 we shall for simplicity restrict our presentation to the case $m=2$, and we assume that $f(0,0)=0$. Some of the examples are primarily of theoretical interest whereas others have practical relevance.

Let $U_{i}=U_{i 1}, V_{i}=U_{i 2}, X=X_{1}=\sum_{i=1}^{N} U_{i}$, and $Y=X_{2}=\sum_{i=1}^{N} V_{i}$. In this case (3.4) gives

$$
\begin{array}{r}
g(x, y)=\sum_{u=0}^{x}\left(a+b \frac{u}{x}\right) \sum_{v=0}^{y} f(u, v) g(x-u, y-v) \\
(x=1,2, \ldots ; y=0,1, \ldots) \\
g(x, y)=\sum_{v=0}^{y}\left(a+b \frac{v}{y}\right) \sum_{\substack{u=0 \\
(x=0,1, \ldots ; y=1,2, \ldots)}}^{x} f(u, v) g(x-u, y-v),
\end{array}
$$

and from (3.8) we obtain

$$
g(x, y)=\sum_{u=0}^{x} \sum_{v=0}^{y}\left(a+b \frac{u+v}{x+y}\right) f(u, v) g(x-u, y-v) . \quad((x, y) \neq(0,0))
$$

Notice that, unlike in (3.4), in these formulae we have included $(u, v)=(0,0)$ in the summations to simplify the display of the formulae. However, as by assumption $f(0,0)=0$, the extra term is equal to zero.

If we were to base our evaluation of $g(x, y)$ on (4.1) for all $(x, y)$ 's such that $x>0$, then we could evaluate $g(0, y)$ by (4.2), that is,

$$
g(0, y)=\sum_{v=1}^{y}\left(a+b \frac{v}{y}\right) f(0, v) g(0, y-v) . \quad(y=1,2, \ldots)
$$


However, if $U_{i}$ is always positive, then together with $g(0,0)$ the recursion (4.1) specifies $g$ completely. In this case we have

$$
g(0, y)=0 . \quad(y=1,2, \ldots)
$$

4C. To assume that $U_{i}$ and $V_{i}$ are independent does not seem to bring any substantial simplification to our recursions. In that case, if $h$ and $k$ denote the marginal probability functions of $U_{i}$ and $V_{i}$, then we can write (4.1) as

$$
\begin{array}{r}
g(x, y)=\sum_{u=0}^{x}\left(a+b \frac{u}{x}\right) h(u) \sum_{v=0}^{y} k(v) g(x-u, y-v) . \\
(x=1,2, \ldots ; y=0,1, \ldots)
\end{array}
$$

4D. Let us now consider the situation with two types of claims. Unlike in subsection 4A we do not exclude the possibility that one claim event can induce payments of both types. We let $U_{i}=1$ if claim event $i$ leads to payments of type 1 , and $U_{i}=0$ if that is not the case. Analogously we let $V_{i}=1$ if claim event $i$ leads to payments of type 2 , and $V_{i}=0$ if that is not the case. Then $X$ and $Y$ are the total numbers of claims of type 1 and 2 respectively. In this case $f(u, v)$ can be positive only when $u, v \in\{0,1\}$, and (4.1) reduces to

$$
\begin{aligned}
& g(x, y)=\left(a+\frac{b}{x}\right)(f(1,0) g(x-1, y)+f(1,1) g(x-1, y-1))+ \\
& \text { af }(0,1) g(x, y-1) . \quad(x=1,2, \ldots ; y=0,1, \ldots)
\end{aligned}
$$

4E. We now leave the restriction that $U_{i}$ and $V_{i}$ can only take the values 0 and 1 , and consider the case when $\mathrm{E} U_{i} V_{i}=0$. As pointed out in subsection $2 \mathrm{C}$, in the situation with different types of payments, this corresponds to the case that a claim cannot have payments of more than one type. Let $c$ denote the probability that a claim is of type $1, h$ the conditional probability function of the claim amount given that the claim is of type 1 , and $k$ the conditional probability function of the claim amount given that the claim is of type 2 . As $f(0,0)$ should be equal to zero, we have that $h(0)=k(0)=0$. Then

$$
f(u, v)= \begin{cases}\operatorname{ch}(u) & (u=1,2, \ldots ; v=0) \\ (1-c) k(v) & (u=0 ; v=1,2, \ldots) \\ 0 . & \text { otherwise }\end{cases}
$$


Insertion in (4.1) gives

$$
\begin{gathered}
g(x, y)=c \sum_{u=1}^{x}\left(a+b \frac{u}{x}\right) h(u) g(x-u, y)+(1-c) a \sum_{v=1}^{y} k(v) g(x, y-v) . \\
(x=1,2, \ldots ; y=0,1, \ldots)
\end{gathered}
$$

In the special case with $a=0$, that is, $N$ is Poisson distributed with parameter $b$, the last summation in (4.5) vanishes, and we obtain

$$
g(x, y)=\frac{c b}{x} \sum_{u=1}^{x} h(u) g(x-u, y) . \quad(x=1,2, \ldots ; y=0,1, \ldots)
$$

As pointed out in subsection $2 \mathrm{C}$, in the present case we know that $X$ and $Y$ are independent. Thus $g(x, y)=s(x) t(y)$, where $s$ and $t$ denote the marginal probability functions of respectively $X$ and $Y$. Insertion in (4.6) gives

$$
s(x) t(y)=t(y) \frac{c b}{x} \sum_{u=1}^{x} h(u) s(x-u) . \quad(x=1,2, \ldots ; y=0,1, \ldots)
$$

As there must exist some $y$ such that $t(y)>0$, we obtain

$$
s(x)=\frac{c b}{x} \sum_{u=1}^{x} h(u) s(x-u) . \quad(x=1,2, \ldots)
$$

This is the univariate Panjer recursion for a compound Poisson distribution with Poisson parameter $c b$ and severity distribution with probability function $h$. As

$$
s(x)=\sum_{y=0}^{\infty} g(x, y), \quad(x=1,2, \ldots)
$$

we could also have obtained (4.7) from (4.6) by summation over $y$.

If we, leaving the Poisson assumption, define $U_{i}$ and $V_{i}$ as in subsection 4D, we obtain

$$
f(1,0)=c ; \quad f(0,1)=1-c ; \quad f(1,1)=0,
$$

and insertion in (4.4) gives

$$
\begin{array}{r}
g(x, y)=\left(a+\frac{b}{x}\right) \operatorname{cg}(x-1, y)+a(1-c) g(x, y-1) . \\
(x=1,2, \ldots ; y=0,1, \ldots)
\end{array}
$$


$4 \mathrm{~F}$. The model of subsection $4 \mathrm{E}$ can also be expressed within the framework of subsection 1B. For $j=1,2$ let $N_{j}$ be the number of claims of type $j$ and $W_{i j}$ the amount of the $i$ th of these claims. Then the assumptions of subsection $1 \mathrm{~B}$ are fulfilled with $f_{1}=h$ and $f_{2}=k$.

The conditional distribution of $N_{1}$ given that $N=n$, is binomial with

$$
\begin{array}{r}
\operatorname{Pr}\left(N_{1}=n_{1} \mid N=n\right)=\left(\begin{array}{c}
n \\
n_{1}
\end{array}\right) c^{n_{1}}(1-c)^{n-n_{1}} . \\
\left(n_{1}=0,1, \ldots, n ; n=0,1, \ldots\right)
\end{array}
$$

Thus for $n_{1}, n_{2}=0,1, .$. we have

$$
\begin{aligned}
& q\left(n_{1}, n_{2}\right)=\operatorname{Pr}\left(\left(N_{1}=n_{1}\right) \cap\left(N_{2}=n_{2}\right)\right)= \\
& \operatorname{Pr}\left(\left(N_{1}=n_{1}\right) \cap\left(N=n_{1}+n_{2}\right)\right)= \\
& \operatorname{Pr}\left(N=n_{1}+n_{2}\right) \operatorname{Pr}\left(N_{1}=n_{1} \mid N=n_{1}+n_{2}\right)= \\
& p\left(n_{1}+n_{2}\right)\left(\begin{array}{c}
n_{1}+n_{2} \\
n_{1}
\end{array}\right) c^{n_{1}}(1-c)^{n_{2}} .
\end{aligned}
$$

We are now within Model A of Hesselager (1996), and the recursions (4.5) and (4.8) are given in his Theorems 2.2 and 2.1 respectively.

$4 \mathrm{G}$. Let us now consider a univariate situation. Let $W_{i}$ be the amount of the $i$ th claim. We assume that the $W_{i}$ 's are positive, integer-valued, mutually independent and identically distributed with common probability function $h$, and independent of $N$. Let $s$ be a positive integer. We say that a claim is of type 1 if it is less than or equal to $s$, and of type 2 if it is greater than $s$. In this case we have

$$
g(x, y)=0 . \quad(x=0,1, \ldots ; y=1,2, \ldots, s)
$$

We now have a special case of the situation of subsection $4 \mathrm{E}$ with

$$
f(u, v)= \begin{cases}h(u) & (u=1,2, \ldots, s ; v=0) \\ h(v) & (u=0 ; v=s+1, s+2, \ldots) \\ 0, & \text { otherwise }\end{cases}
$$

and insertion in (4.1) gives

$$
\begin{array}{r}
g(x, 0)=\sum_{u=1}^{s}\left(a+b \frac{u}{x}\right) h(u) g(x-u, 0) \quad(x=1,2, \ldots) \\
g(x, y)=\sum_{u=1}^{s}\left(a+b \frac{u}{x}\right) h(u) g(x-u, y)+a \sum_{v=s+1}^{y} h(v) g(x, y-v) ; \\
(x=1,2, \ldots ; y=s+1, s+2, \ldots)
\end{array}
$$


Insertion of (4.9) in (4.2) gives

$$
\begin{array}{r}
g(x, y)=a \sum_{u=1}^{s} h(u) g(x-u, y)+\sum_{v=s+1}^{y}\left(a+b \frac{v}{y}\right) h(v) g(x, y-v) . \\
(x=0,1, \ldots ; y=s+1, s+2, \ldots)
\end{array}
$$

$4 \mathrm{H}$. In the previous subsection, we distinguished between claims less than or equal to $s$ and claims larger than $s$. A more interesting situation would be to let

$$
U_{i}=\min \left(W_{i}, s\right) ; \quad V_{i}=\max \left(W_{i}-s, 0\right) .
$$

Under an unlimited excess-of-loss treaty with retention $s, U_{i}$ and $V_{i}$ are respectively the retained and reinsured parts of the $i$ th claim. Thus $X$ becomes the total payments of the ceding company and $Y$ the total payments of the reinsurer. In this case we have

$$
g(x, y)=0 . \quad(x=0,1, \ldots, s-1 ; y=1,2, \ldots)
$$

Analogous to (4.9) we obtain

$$
f(u, v)= \begin{cases}h(u) & (u=1,2, \ldots, s ; v=0) \\ h(v+s) & (u=s ; v=1,2, \ldots) \\ 0 . & \text { otherwise }\end{cases}
$$

Insertion in (4.1) gives

$$
\begin{aligned}
& g(x, y)=\sum_{u=1}^{s}\left(a+b \frac{u}{x}\right) h(u)(g(x-u, y)+ \\
& \left(a+b \frac{s}{x}\right) \sum_{v=1}^{y} h(v+s) g(x-s, y-v) . \\
& (x=1,2, \ldots ; y=0,1, \ldots)
\end{aligned}
$$

As now $U_{i}$ is always positive, together with $g(0,0)$ this recursion completely specifies $g$.

Insertion of (4.10) in (4.2) gives

$$
\begin{array}{r}
g(x, y)=a \sum_{u=1}^{s} h(u) g(x-u, y)+\sum_{v=1}^{y}\left(a+b \frac{v}{y}\right) h(v+s) g(x-s, y-v) . \\
(x=1,2, \ldots ; y=1,2, \ldots)
\end{array}
$$

For small $y$ this recursion may be more convenient than (4.11). However, we will still need (4.11) to evaluate $g(x, 0)$. 
4I. Let us finally consider the case when $U_{i}=W_{i}$ and $V_{i}=1$. Then $X$ will be the aggregate claims and $Y$ the number of claims, that is, $g$ will be the joint probability function of the aggregate claims and the number of claims. Thus we have

$$
g(x, y)=\operatorname{Pr}(Y=y) \operatorname{Pr}(X=x \mid Y=y)=p(y) h^{y *}(x) . \quad(x, y=0,1, \ldots)
$$

Furthermore,

$$
f(u, v)= \begin{cases}h(u) & (u=1,2, \ldots, s ; v=1) \\ 0, & \text { otherwise }\end{cases}
$$

and insertion in respectively (4.1) and (4.2) gives

$$
\begin{aligned}
& g(x, y)=\sum_{u=1}^{x}\left(a+b \frac{u}{x}\right) h(u) g(x-u, y-1) \quad(x, y=1,2, \ldots) \\
& g(x, y)=\left(a+\frac{b}{y}\right) \sum_{u=1}^{x} h(u) g(x-u, y-1) . \quad(x, y=1,2, \ldots)
\end{aligned}
$$

As both $U_{i}$ and $V_{i}$ are always positive, together with $g(0,0)$ each of the recursions completely specifies $g$.

The recursion (4.14) can easily be seen more directly. By successively applying (4.12) and (1.2) in the right-hand side of (4.14) we obtain

$$
\begin{aligned}
& \left(a+\frac{b}{y}\right) \sum_{u=1}^{x} h(u) g(x-u, y-1)= \\
& \left(a+\frac{b}{y}\right) \sum_{u=1}^{x} h(u) p(y-1) h^{(y-1) *}(x-u)= \\
& \left(a+\frac{b}{y}\right) p(y-1) \sum_{u=1}^{x} h(u) h^{(y-1) *}(x-u)=p(y) h^{y *}(x)=g(x) .
\end{aligned}
$$

It does not seem possible to give such a simple interpretation of (4.13).

\section{Convolutions}

5A. Let us now consider the special case of Theorem 1 when $p$ is the binomial probability function

$$
p(n)=\left(\begin{array}{l}
r \\
n
\end{array}\right) c^{n}(1-c)^{r-n} . \quad(n=0,1, \ldots, r ; r=1,2, \ldots ; 0<c<1)
$$


Then

$$
a=-\frac{c}{1-c} ; \quad b=(r+1) \frac{c}{1-c} .
$$

We assume that $f(\mathbf{0})=0$. Application of Theorem 1 gives

$$
\begin{gathered}
g(\mathbf{0})=(1-c)^{r} \\
g(\mathbf{x})=\frac{c}{1-c} \sum_{\mathbf{0}<\mathbf{u} \leq \mathbf{x}}\left((r+1) \frac{u_{k}}{x_{k}}-1\right) f(\mathbf{u}) g(\mathbf{x}-\mathbf{u}) . \quad\left(\mathbf{x} \geq \mathbf{e}_{k}\right)
\end{gathered}
$$

We shall apply this recursion to deduce a multivariate extension of De Pril's (1985) recursion for convolutions.

5B. Let $\mathbf{V}_{1}, \ldots, \mathbf{V}_{r}$ be $r m$-dimensional independent and identically distributed random vectors with non-negative integer-valued elements and common probability function $h$. It is assumed that $0<h(\mathbf{0})<1$. We want to deduce a recursion for the probability function $g$ of $\mathbf{X}=\sum_{i=1}^{r} \mathbf{V}_{i}$. We obviously have $g=h^{r *}$.

Let

$$
\begin{gathered}
c=1-h(\mathbf{0}) \\
f(\mathbf{y})=\frac{h(\mathbf{y})}{c} . \quad(\mathbf{y}>0)
\end{gathered}
$$

The function $f$ can be interpreted as the conditional probability function of $\mathbf{V}_{i}$ given that at least one of its elements is greater than zero. We now have that $\mathbf{X}$ has a compound binomial distribution with counting distribution given by (5.1) with $c$ given by (5.4) and severity distribution with probability function $f$. Insertion of (5.4) and (5.5) in (5.2) and (5.3) gives

$$
\begin{gathered}
g(\mathbf{0})=h(\mathbf{0})^{r} \\
g(\mathbf{x})=\frac{1}{h(\mathbf{0})} \sum_{\mathbf{0}<\mathbf{u} \leq \mathbf{x}}\left((r+1) \frac{u_{k}}{x_{k}}-1\right) h(\mathbf{u}) g(\mathbf{x}-\mathbf{u}) . \quad\left(\mathbf{x} \geq \mathbf{e}_{k}\right)
\end{gathered}
$$

Analogously, by application of (3.8) we obtain

$$
g(\mathbf{x})=\frac{1}{h(0)} \sum_{0<\mathbf{u} \leq \mathbf{x}}\left((r+1) \frac{u .}{x .}-1\right) h(\mathbf{u}) g(\mathbf{x}-\mathbf{u}) . \quad(\mathbf{x}>0)
$$


When $m=1$, both these recursions reduce to

$$
\begin{gathered}
g(\mathbf{0})=h(\mathbf{0})^{r} \\
g(x)=\frac{1}{h(0)} \sum_{u=1}^{x}\left((r+1) \frac{u}{x}-1\right) h(u) g(x-u) . \quad(x=1,2, \ldots)
\end{gathered}
$$

This is the recursion deduced by De Pril (1985).

For $j=1, \ldots, m$, let $h_{j}$ and $g_{j}$ denote the marginal probability functions of $V_{1 j}$ and $X_{j}$ respectively. As $h(0)>0$, we must have $h_{j}(0)>0$ for $j=1, \ldots, m$. If $V_{11}, \ldots, V_{1 m}$ are independent, then $X_{1}, \ldots, X_{m}$ are also independent, and we have

$$
h(\mathbf{u})=\prod_{j=1}^{m} h_{j}\left(u_{j}\right) ; \quad g(\mathbf{x})=\prod_{j=1}^{m} g_{j}\left(x_{j}\right) .
$$

Insertion in (5.6) gives

$$
\prod_{j=1}^{m} g_{j}\left(x_{j}\right)=\sum_{0<\mathbf{u} \leq \mathbf{x}}\left((r+1) \frac{u_{k}}{x_{k}}-1\right) \prod_{j=1}^{m} \frac{h_{j}\left(u_{j}\right) g_{j}\left(x_{j}-u_{j}\right)}{h_{j}(0)} . \quad\left(\mathbf{x} \geq \mathbf{e}_{k}\right)
$$

However, in this case it seems more convenient to evaluate each of the $g_{j}$ 's separately by the univariate recursion (5.7) and then multiply them.

If $\prod_{j \neq k} g_{j}\left(x_{j}\right)>0$, we can rewrite (5.8) as

$$
\begin{aligned}
& g_{k}\left(x_{k}\right)== \\
& \frac{1}{h_{k}(0)} \sum_{\mathbf{0}<\mathbf{u} \leq \mathbf{x}}\left((1+r) \frac{u_{k}}{x_{k}}-1\right) h_{k}\left(u_{k}\right) g_{k}\left(x_{k}-u_{k}\right) \prod_{j \neq k} \frac{h_{j}\left(u_{j}\right) g_{j}\left(x_{j}-u_{j}\right)}{h_{j}(0) g_{j}\left(x_{j}\right)} . \quad\left(\mathbf{x} \geq \mathbf{e}_{k}\right)
\end{aligned}
$$

As the univariate recursion (5.7) gives

$$
\begin{array}{r}
g_{k}\left(x_{k}\right)=\frac{1}{h_{k}(0)} \sum_{u_{k}=1}^{x_{k}}\left((r+1) \frac{u_{k}}{x_{k}}-1\right) h_{k}\left(u_{k}\right) g_{k}\left(x_{k}-u_{k}\right), \\
\left(x_{k}=1,2, \ldots\right)
\end{array}
$$

it is tempting to conclude that we obtain 1 by a summation over the $u_{j}$ 's of

$$
\prod_{j \neq k} \frac{h_{j}\left(u_{j}\right) g_{j}\left(x_{j}-u_{j}\right)}{h_{j}(0) g_{j}\left(x_{j}\right)}
$$

However, in the next subsection we shall see that it is not that simple. 
5C. Let us now consider the special case $m=2$. In that case (5.6) with $k=1$ gives

$$
\begin{aligned}
& g(x, y)=\frac{1}{h(0,0)}\left[\sum_{u=1}^{x}\left((r+1) \frac{u}{x}-1\right) \sum_{v=o}^{y} h(u, v) g(x-u, y-v)-\right. \\
& \left.\sum_{v=1}^{y} h(0, v) g(x, y-v)\right] ; \quad(x=1,2, \ldots ; y=0,1, \ldots)
\end{aligned}
$$

as $h(0,0)>0$, we cannot include $(u, v)=(0,0)$ in the summation as in (4.1) and (4.2).

If $V_{11}$ and $V_{12}$ are independent, (5.10) gives that for $x=1,2, \ldots ; y=0,1, \ldots$

$$
\begin{aligned}
& g_{1}(x) g_{2}(y)=\frac{1}{h_{1}(0) h_{2}(0)} \times \\
& {\left[\left(\sum_{u=1}^{x}\left((r+1) \frac{u}{x}-1\right) h_{1}(u) g_{1}(x-u)\right) \sum_{v=0}^{y} h_{2}(v) g_{2}(y-v)-\right.} \\
& \left.h_{1}(0) g_{1}(x) \sum_{v=1}^{y} h_{2}(v) g_{2}(y-v)\right]
\end{aligned}
$$

that is,

$$
\begin{aligned}
& g_{1}(x) g_{2}(y)= \\
& \frac{1}{h_{1}(0) h_{2}(0)}\left[A \sum_{v=0}^{y} h_{2}(v) g_{2}(y-v)+h_{1}(0) g_{1}(x) h_{2}(0) g_{2}(y)\right]
\end{aligned}
$$

with

$$
A=\sum_{u=1}^{x}\left((r+1) \frac{u}{x}-1\right) h_{1}(u) g_{1}(x-u)-h_{1}(0) g_{1}(x)
$$

From (5.9) we see that $A=0$, and thus the right-hand expression in (5.11) reduces to $g_{1}(x) g_{2}(y)$.

\section{ACKNOWLEDGEMENT}

The present research was carried out while the author stayed as GIO Visiting Professor at the Centre for Actuarial Studies, University of Melbourne. 


\section{REFERENCES}

De PrIL, N. (1985). Recursions for convolutions of arithmetic distributions. ASTIN Bulletin 15, 135-139.

HeSSELAGER, O. (1996). Recursions for certain bivariate counting distributions and their compound distributions. ASTIN Bulletin 26, 35-52.

PANJER, H.H. (1981). Recursive evaluation of a family of compound distributions. ASTIN Bulletin 12, 22-26.

SunDt, B. (1992). On some extensions of Panjer's class of counting distributions. ASTIN Bulletin 22, 61-80.

SUNDT, B. (1993). An introduction to non-life insurance mathematics. (3. ed.) Verlag Versicherungswirtschaft e.V., Karlsruhe.

SundT, B. (1998). The multivariate De Pril transform. Research paper 59, Centre for Actuarial Studies, University of Melbourne.

BJøRn SUNDT

Department of Mathematics

University of Bergen

Johannes Bruns gate 12

N-5008 Bergen

Norway 